\title{
IMPACTO DA EXPOSIÇÃO AO AMIANTO NA SAÚDE DA POPULAÇÃO ADULTA: REVISÃO INTEGRATIVA
}

\author{
Fátima Denise Padilha Baran ${ }^{1}$, Lara Adrianne Garcia Paiano ${ }^{1}$, Nen Nalú Alves das Mercês ${ }^{2}$
}

\begin{abstract}
RESUMO: Objetivou-se identificar o impacto da exposição ao amianto na saúde da população adulta, nas publicações científicas. Revisão Integrativa de literatura realizada em bases de dados na área da saúde no período de 2005 a 2015. Foram selecionados 15 estudos. Verificou-se o impacto da exposição ao amianto na população adulta com o adoecimento por mesotelioma maligno, asbestose, câncer de pulmão, entre outros; a mortalidade por mesotelioma em trabalhadores conferiu prevalência significativa para o estudo. A taxa mortalidade é preocupante, haja visto que as possibilidades terapêuticas são limitadas. O impacto do amianto ultrapassa os aspectos biológicos, trazendo alterações psicoemocionais significativas na saúde da população. Destaca-se a importância do debate sobre o impacto do amianto na saúde do adulto, principalmente no campo da saúde do trabalhador sobre a necessidade do banimento de seu uso nos países que o mantém em escala industrial e comercial.
\end{abstract}

DESCRITORES: Asbestos; Mesotelioma; Adultos; Risco ocupacional; Saúde do trabalhador.

\section{IMPACT OF ASBESTOS EXPOSURE ON THE HEALTH OF THE ADULT POPULATION: AN INTEGRATIVE REVIEW}

ABSTRACT: The objective of this study was to identify the impact of asbestos exposure on the health of the adult population in scientific publications. An integrative literature review was carried out in health databases in the period from 2005 to 2015. Fifteen studies were selected.The impact of asbestos exposure on the adult populationwas observed inthe onset of diseases such as malignant mesothelioma, asbestosis, and lung cancer.Mortality due to mesothelioma in workershad a significant prevalence for the study. Mortality ratesare alarming, considering that the therapeutic possibilities are limited. The impact of asbestos exposure surpasses biological aspects, bringing significant psycho-emotional changes to the health of the population. Therefore, it is important to discuss the asbestos impact on adult health, primarily in worker's health, regarding the need for banning its use in countries that still use it in industrial and commercial scale.

DESCRIPTORS: Asbestos; Mesothelioma; Adults; Occupational risks; Worker's health.

\section{IMPACTO DE LA EXPOSICIÓN AL AMIANTO EN LA SALUD DE LA POBLACIÓN ADULTA: REVISIÓN INTEGRATIVA}

RESUMEN: Se objetivó identificar el impacto de la exposición al amianto en la salud de la población adulta, en las publicaciones científicas. Revisión integrativa de literatura realizada en bases de datos del área de salud entre 2005 y 2015 . Fueron seleccionados 15 estudios. Se verificó el impacto de exposición al amianto en población adulta con padecimiento de mesotelioma maligno, asbestosis cáncer de pulmón, etc.; la mortalidad por mesotelioma en trabajadores le confirió prevalencia significativa al estudio. La tasa de mortalidad es preocupante, visto que las posibilidades terapéuticas son limitadas. El impacto del amianto sobrepasa los aspectos biológicos, provocando alteraciones psicoemocionales significativas en la salud de la población. Se destaca la importancia del debate sobre impacto del amianto en la salud del adulto, especialmente en el área de salud del trabajador, enfatizando la necesidad de la prohibición de su utilización en los países que lo mantienen en escala industrial y comercial.

DESCRIPTORES: Asbestos; Mesotelioma; Adultos; Riesgos Laborales; Salud Laboral.

${ }^{1}$ Enfermeira. Mestranda em Enfermagem. Universidade Federal do Paraná. Curitiba, PR, Brasil.

${ }^{2}$ Enfermeira. Doutora em Enfermagem. Docente de Enfermagem da Universidade Federal do Paraná. Curitiba, PR, Brasil.

Autor Correspondente:

Nen Nalu Alves das Mercês

Universidade Federal do Paraná

Av. Prefeito Lothário Meissner, 3400 - 80210-170 - Curitiba, PR, Brasil

E-mail: nennalu@gmail.com
Recebido: $19 / 05 / 2016$

Finalizado: 03/06/2016 


\section{- INTRODUÇÃO}

O amianto é um mineral amplamente utilizado ao redor do mundo durante décadas, principalmente entre os anos de 1950 e 1970, na fabricação de produtos, tais como: fibrocimento para a produção de caixas, telhas; lonas de freios de automóveis, entre outros ${ }^{(1)}$. O mineral é classificado em seis variações: serpentina (crisotila - amianto branco), anfibólios (amosita, actinolita - amianto marrom), e antofilita, crocidolite e tremolita (amianto azul) ${ }^{(2)}$.

As primeiras menções da potencialidade nociva do amianto aos seres humanos ocorreram entre as décadas de 50 e $60^{(3-4)}$, com o resultado dos estudos sobre os mecanismos de ação do amianto prejudiciais à saúde humana ${ }^{(5)}$. Mas foi no final da década de 70 que a International Agency Research of Cancer (IARC), em parceria com a Organização Mundial da Saúde (OMS), confirmaram que a exposição ao amianto é cancerígena a seres humanos ${ }^{(2)}$.

A exposição ocorre pela inalação das fibras dispersas no ar, encontradas em geral no ambiente de trabalho e em proximidades de indústrias que manipulam o mineral ${ }^{(6)}$. Dessa forma, o risco ocupacional é uma preocupação emergente, pois a carga de doenças tem um maior impacto em homens, que trabalham diretamente com o amianto ${ }^{(7-8)}$ e com tempo de exposição em geral superior a 20 anos $^{(9)}$.

Todas as classes de amianto são prejudiciais em qualquer nível de exposição(10), inclusive o crisotila, comercializado em diversos países ${ }^{(11)}$. Os estudos apontam as doenças provocadas pela exposição e o câncer lidera o ranking, entre eles: o de pulmão, mesotelioma pleural, peritoneal, pericárdio (câncer raro e fatal associado em $80 \%$ com a exposição) $)^{(12)}$. Além de doenças pleuropulmonares, como fibrose pulmonar idiopática ${ }^{(13)}$ e asbestose ${ }^{(14)}$.

Cessando hoje a utilização do amianto, continuará nas próximas décadas o surgimento de doenças, devido ao longo período para manifestação dos primeiros sintomas, denominado período de latência ${ }^{(6)}$, a exemplo do mesotelioma maligno ${ }^{(15)}$. Dessa forma, a exposição ao amianto se tornou uma urgência sanitária, pelos riscos eminentes à saúde da população adulta ${ }^{(16)}$.

Portanto, a luta para proibir a sua utilização em qualquer etapa do processo industrial e dos produtos comercializados tornou-se imperiosa ${ }^{(10,17)}$. De acordo com dados da International ban asbestos o amianto foi proibido em 57 países, entretanto cerca de 200 milhões de toneladas continuam sendo extraídas, e os maiores produtores e consumidores são a Rússia, China e Brasil ${ }^{(18)}$.

O presente artigo tem como objetivo identificar, nas publicações cientificas, o impacto da exposição ao amianto na saúde da população adulta.

\section{MÉTODO}

Trata-se de um estudo descritivo, do tipo revisão integrativa de literatura, sobre a produção científica, com relação ao impacto do amianto na saúde. Essa modalidade de revisão permite a inclusão de estudos experimentais e não experimentais para a compreensão de um fenômeno. Combina dados de literatura teórica e empírica, além de incorporar propósitos, suposições, conceitos, revisões, teorias, gerando possibilidades de análises sólidas para colaborar com o conhecimento para a saúde(19).

Para estruturar esta revisão, seguiram-se seis etapas metodológicas: i) seleção da questão norteadora para revisão; ii) estabelecimento de critérios para seleção da amostra; iii) apresentação das características da pesquisa primária; iv) análise dos dados; v) interpretação dos resultados; e, vi) apresentação da revisão(19).

Este estudo foi guiado pela questão norteadora: Qual o impacto da exposição ao amianto na saúde da população adulta?

Os critérios de seleção da amostra foram: ser artigo científico publicado na literatura nacional e internacional, que compreendessem a temática, com textos disponíveis na íntegra online, em periódicos indexados nas bases de dados eletrônicas consultadas, nos anos de 2005 a 2015, em português, inglês e espanhol. Foram excluídos os artigos que não atenderam ao objetivo proposto, aos critérios de seleção 
e que fossem artigos de revisão (integrativa e sistemática).

A busca dos estudos foi executada de forma eletrônica nas seguintes bases de dados: Literatura Latino-americana e do Caribe em Ciências Sociais e da Saúde (LILACS), National Library of Medicine (PubMed), Medical Literature Analysis and Retrieval System on-line (MEDLINE) e Scientific Eletronic Library (SCIELO), no período de fevereiro a março de 2016. Utilizou-se descritores do MESH (Medical Subject Headings): asbestos, mesothelioma, adults, exposure, risk occupational, health workers. Foi utilizado o operador boleano and para a combinação entre eles (Quadro 1).

Para a coleta de dados, foi elaborado pelos pesquisadores um instrumento visando a assegurar a totalidade dos dados relevantes e minimizar erros na transcrição; contendo identificação (autoria, ano de publicação e país), objetivo do estudo, delineamento, participantes, nível de evidência e desfecho.

O nível de evidência foi classificado de acordo com a categorização da Agency for Health care Research and Quality (AHRQ). A qualidade das evidências é classificada em sete níveis: nível I - meta análise e revisões sistemáticas; nível II - estudos individuais/delineamento experimental; nível III evidência de estudo quase experimental; nível IV - estudos descritivos com abordagem qualitativa; nível V - estudos de caso; nível VI - estudos descritivos; nível VII - opinião de especialistas ${ }^{(20)}$.

A seleção das publicações foi realizada por duas revisoras de forma independente. A seguir o fluxograma do processo de seleção dos artigos que compuseram a amostra, baseado nos critérios do Preferred Reporting Items for Systematic Review and a Meta-Analyses (PRISMA) (Figura 1).

Quadro 1 - Resultados da busca eletrônica nas bases de dados pesquisadas. Curitiba, PR, Brasil, 2016

\begin{tabular}{|l|c|c|c|c|}
\hline Descritores & LILACS & MEDLINE & PubMed & SCIELO \\
\hline Asbestos and exposure and adults & 1 & 12 & 332 & 13 \\
\hline Mesothelioma and asbestos and ocupacional risk & 0 & 74 & 87 & 0 \\
\hline Asbestos and health workers & 0 & 26 & 160 & 16 \\
\hline
\end{tabular}

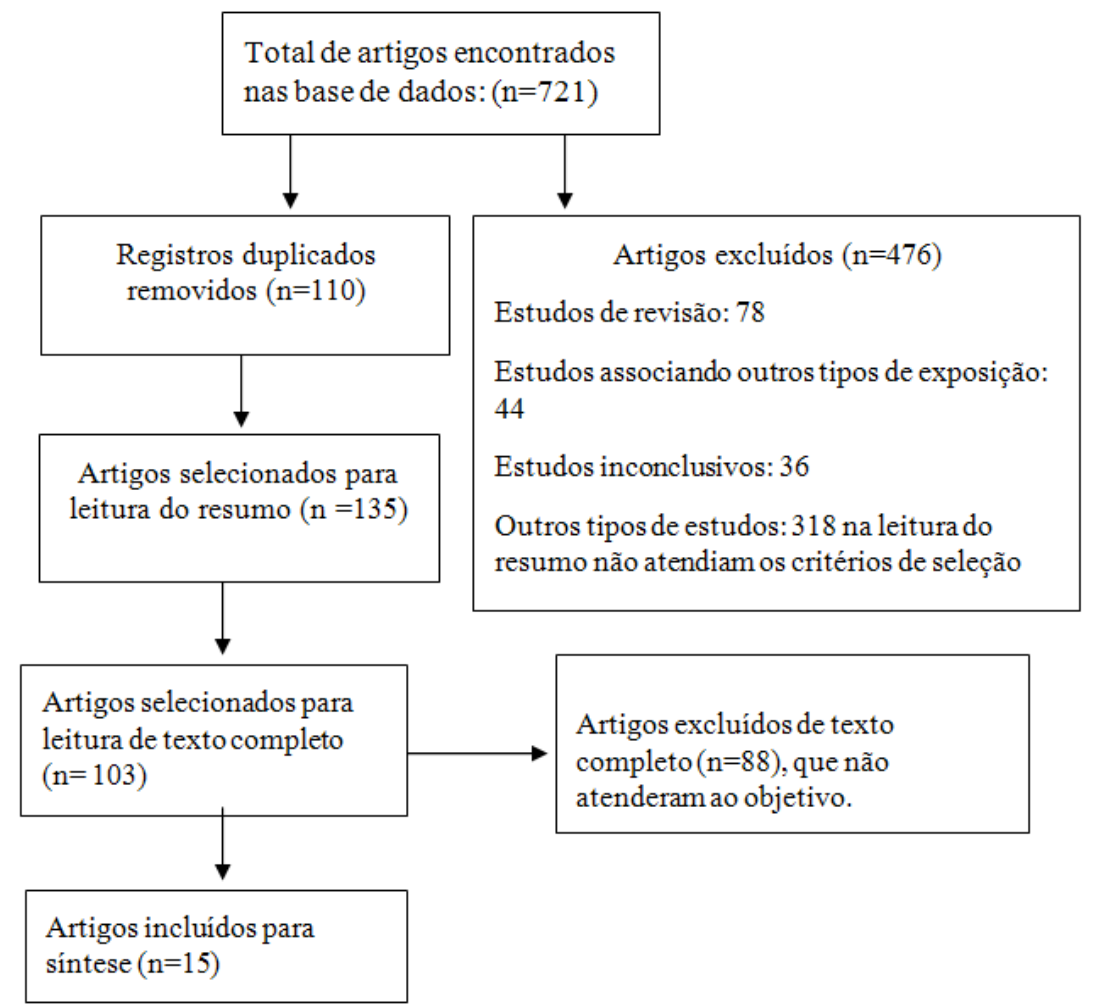

Figura 1 - Fluxograma da seleção dos artigos científicos que compuseram a amostra da revisão integrativa. Curitiba, PR, Brasil, 2016 


\section{RESUlTADOS}

Os estudos selecionados para a síntese encontravam-se na PubMed(14) e um no SCIELO correspondendo a amostra. Quanto ao país de publicação dos estudos, cinco foram na Inglaterra/ Reino Unido, quatro nos Estados Unidos e um respectivamente na Argentina, Itália, Japão, Espanha, Suíça e Brasil. Quanto ao idioma, todos os estudos publicados na língua inglesa, sendo dois também em espanhol, dois em italiano e um em português. O ano de publicação variou de 2005 a 2015: um de 2015, quatro de 2013, dois de 2012, três de 2011, um de 2010, em de 2007, e três de 2005. Quanto ao nível de evidências, sete estudos de nível IV e oito de nível VI . No quadro 2 são apresentados a caracterização dos estudos/artigos.

Quadro 2 - Caracterização dos artigos publicados no período de 2005 a 2015, na PubMed e SCIELO. Curitiba, PR, Brasil, 2016.

\begin{tabular}{|c|c|c|c|}
\hline $\begin{array}{l}\text { Autoria } \\
\text { Ano e País de } \\
\text { publicação }\end{array}$ & $\begin{array}{l}\text { Objetivo/Delineamento do estudo/ } \\
\mathrm{N}=\text { participantes }\end{array}$ & $\begin{array}{c}\text { Nível de } \\
\text { Evidência }\end{array}$ & Desfecho \\
\hline $\begin{array}{l}\text { 1. Abente GL, } \\
\text { Gómez MG, } \\
\text { Navarro AM, } \\
\text { Navarro PF, } \\
\text { Ramis R, Perez } \\
\text { JG, et al (21). } \\
\text { 2013/ Inglaterra }\end{array}$ & $\begin{array}{l}\text { Objetivo: estudar as tendências de } \\
\text { mortalidade por câncer pleural como } \\
\text { um indicador de mortalidade do } \\
\text { mesotelioma com previsões até o ano } \\
\text { de } 2020 . \\
\text { Delineamento: Estudo descritivo } \\
\text { Prospectivo. }\end{array}$ & VI & $\begin{array}{l}\text { A tendência de mortes por mesotelioma } \\
\text { e câncer de pleura relacionado com } \\
\text { o trabalho continuarão a ocorrer na } \\
\text { Espanha pelo menos até o ano de } 2040 \text {. }\end{array}$ \\
\hline $\begin{array}{l}\text { 2. Zurbriggen R, } \\
\text { Capone L.(22) } \\
\text { 2013/ Argentina }\end{array}$ & $\begin{array}{l}\text { Objetivo: Descrever as manifestações } \\
\text { clínicas de alterações pulmonares } \\
\text { dos trabalhadores de uma empresa } \\
\text { de aço. } \\
\text { Delineamento: Estudo clínico } \\
\text { Retrospectivo } \\
\mathrm{N}=27\end{array}$ & $\mathrm{VI}$ & $\begin{array}{l}\text { Foram encontradas: } \\
\text { Patologias Benignas: } 16 \text { com } \\
\text { lesões pleuraise } 06 \text { com asbestose. } \\
\text { Patologias malignas: } 4 \\
\text { com mesotelioma e } 01 \\
\text { com carcinoma de pulmão }\end{array}$ \\
\hline $\begin{array}{l}\text { 3. Menegozzo } \\
\text { S, Comba } \\
\text { P, Ferrante } \\
\text { D, Santis M, } \\
\text { Gorini G, Izzo F, } \\
\text { et al. }{ }^{(23)} \\
2011 / \text { Itália } \\
\end{array}$ & $\begin{array}{l}\text { Objetivo: Investigar as causas de } \\
\text { mortalidade entre pessoas do sexo } \\
\text { masculino expostos ao amianto em } \\
\text { uma empresa Italiana } \\
\text { Delineamento: Estudo de Coorte } \\
\mathrm{N}=1247\end{array}$ & IV & \begin{tabular}{|l} 
Causa de mortalidade: \\
Pneuconiose: 42 mortes \\
Asbestose: 41 mortes \\
mesotelioma pleural: 24 mortes \\
câncer de pulmão: 84 mortes \\
câncer peritoneal: 9 mortes \\
câncer de reto: 6 mortes \\
\end{tabular} \\
\hline $\begin{array}{l}\text { 4. Wang X, Yano } \\
\text { E, Lin S, Yu ITS, } \\
\text { Lan Y, Tse LA,et } \\
\text { al. }{ }^{(24)} \\
\text { 2013/ EUA }\end{array}$ & $\begin{array}{l}\text { Objetivo: Avaliar a relação de } \\
\text { mortalidade por Câncer de pulmão } \\
\text { e outras causas relacionadas com } \\
\text { exposição ao amianto. } \\
\text { Delineamento: Estudo de } \\
\text { Coorte realizado na China } \\
\mathrm{N}=1539\end{array}$ & IV & $\begin{array}{l}\text { Aumento da mortalidade por câncer de } \\
\text { pulmão: } \\
\text { Em } 10 \text { anos } 3,5 \text { vezes } \\
\text { Em } 20 \text { anos 5,3 vezes } \\
\text { Na tendência observada para doenças } \\
\text { respiratórias não malignas os fumantes } \\
\text { tiveram maior mortalidade por todas as } \\
\text { causas do que não fumantes. } \\
\text { Foi observada a Relação clara e } \\
\text { direta a exposição ao amianto para o } \\
\text { desenvolvimento de câncer de pulmão } \\
\text { associado a mortalidade }\end{array}$ \\
\hline $\begin{array}{l}\text { 5. Myogin } \\
\text { T, Azuma K, } \\
\text { Okumura J, } \\
\text { Uchiyama I(25) } \\
\text { 2012/ Japão }\end{array}$ & $\begin{array}{l}\text { Objetivo: Descrever a tendência } \\
\text { de mortalidade por mesotelioma } \\
\text { prospectivamente no Japão de } 2003 \\
\text { a } 2050 \\
\text { Delineamento: Estudo } \\
\text { Epidemiológico descritivo. }\end{array}$ & VI & $\begin{array}{l}\text { A tendência de mortalidade continuará } \\
\text { crescendo previsto para o ano de } 2027 \text { e } \\
\text { alcançará o máximo de } 66.327 \text { mortes } \\
\text { entre os anos de } 2003 \text { a } 2050 \text { no Japão }\end{array}$ \\
\hline
\end{tabular}




\begin{tabular}{|c|c|c|c|}
\hline $\begin{array}{l}\text { 6. Pira E, } \\
\text { Pelucchi } \\
\text { C, Buffoni } \\
\text { L, Palmas A, } \\
\text { Turbiglio M, } \\
\text { Negri E, et al. }{ }^{(26)} \\
\text { 2005/Reino } \\
\text { Unido }\end{array}$ & $\begin{array}{l}\text { Objetivo: Analisar os dados de } \\
\text { estudo de Coorte de } 889 \text { homens e } \\
1077 \text { mulheres empregadas por pelo } \\
\text { menos um mês em uma empresa de } \\
\text { amianto. } \\
\text { Delineamento: Estudo de coorte } \\
\mathrm{N}=889 \text { homens } \\
1077=\text { mulheres }\end{array}$ & IV & $\begin{array}{l}\text { Nos trabalhadores italianos estudados: } \\
222 \text { mortes totais sendo atribuídos ao } \\
\text { câncer pleural, peritoneal e pulmão, com } \\
\text { relações diretas das funções ocupacionais } \\
\text { com o amianto para o desenvolvimento } \\
\text { das respectivas doenças. }\end{array}$ \\
\hline $\begin{array}{l}\text { 7. Darnton A, } \\
\text { Hodgson J, } \\
\text { Benson P, } \\
\text { Coggon D. } \\
\text { 2012) } \\
\text { /nglaterra }\end{array}$ & $\begin{array}{l}\text { Objetivo: Analisar em dados } \\
\text { de registros a mortalidade por } \\
\text { mesotelioma e asbestose na Grã } \\
\text { Betanha. } \\
\text { Delineamento: Estudo descritivo em } \\
\text { registro de dados. }\end{array}$ & VI & $\begin{array}{l}\text { Mortalidade de } 1991 \text { a } 2000 \text { : } 33.751 \text { por } \\
\text { mesotelioma e } 5.396 \text { por asbestose. } \\
\text { Taxas de mortalidade por mesotelioma } \\
\text { e asbestose com aumento progressivo } \\
\text { acima dos } 85 \text { anos de idade. } \\
\text { Mortalidade por mesotelioma é maior } \\
\text { entre homens nascidos entre: } 1939 \text { - } \\
\text { 1943. } \\
\text { mortalidade por asbestose em pessoas } \\
\text { nascidas entre } 1924 \text { a } 1938 \text {. }\end{array}$ \\
\hline $\begin{array}{l}\text { 8. Pietro MA, } \\
\text { Suess A, March } \\
\text { JC, Danet A, } \\
\text { Corral OP, } \\
\text { Martin A. }{ }^{(28)} \\
\text { 2011/Espanha }\end{array}$ & $\begin{array}{l}\text { Objetivo: Conhecer as opiniões e } \\
\text { expectativas dos trabalhadores de } \\
\text { uma empresa de Uralita, com doenças } \\
\text { relacionadas a exposição ao amianto. } \\
\text { Delineamento: Estudo qualitativo } \\
\text { realizado por meio da técnica de } \\
\text { Grupo Focal. }\end{array}$ & VI & $\begin{array}{l}\text { Problemas de saúde dos trabalhadores: } \\
\text { asbestose, câncer de pulmão e } \\
\text { mesotelioma. } \\
\text { Opiniões: descontinuidade de atenção } \\
\text { a saúde, dificuldade de diagnóstico } \\
\text { correto, burocracia, escassez de cuidados } \\
\text { específicos para os familiares. } \\
\text { Expectativas: reconhecimento como } \\
\text { enfermidade ocupacional, pagamento } \\
\text { de indenizações, criação de unidades } \\
\text { de atenção especificas com tratamento } \\
\text { humanizado e qualidade de instrumentos } \\
\text { técnicos no sistema de saúde pública e } \\
\text { continuar participando de programas e } \\
\text { protocolos. }\end{array}$ \\
\hline $\begin{array}{l}\text { 9. Cole SR, } \\
\text { Richardson } \\
\text { DB, Haitão C, } \\
\text { Naimi A. }{ }^{(29)} \\
\text { 2013/ EUA }\end{array}$ & $\begin{array}{l}\text { Objetivo: Analisar a mortalidade por } \\
\text { câncer de pulmão em trabalhadores } \\
\text { expostos ao amianto em uma empresa } \\
\text { na Carolina do Sul/EUA } \\
\text { Delineamento: Estudo Coorte } \\
\mathrm{N}=3.002 \text { participantes. }\end{array}$ & IV & $\begin{array}{l}\text { Mortalidade: } 195 \text { por câncer de pulmão, } \\
\text { com alta associação a exposição ao } \\
\text { amianto. }\end{array}$ \\
\hline $\begin{array}{l}10 \text { Hodgson } \\
\text { JT, McElvenny } \\
\text { DM, Darnton } \\
\text { AJ, Price MJ, } \\
\text { Peto J. } \\
\text { 2005/ Inglaterra }\end{array}$ & $\begin{array}{l}\text { Objetivo:Prever a carga de } \\
\text { mortalidade por mesotelioma } \\
\text { futuramente na região da Grã } \\
\text { Bretanha } \\
\begin{array}{l}\text { Delineamento: Estudo descritivo } \\
\text { prospectivo. }\end{array}\end{array}$ & $\mathrm{VI}$ & $\begin{array}{l}\text { Previsão de } 2011 \text { a 2015: } 2.450 \text { mortes/ } \\
\text { ano; } \\
\text { A partir de 2001: } 65.000 \text { mortes } \\
\text { Entre } 1968 \text { a } 2050 \text { haverá cerca de } 90.000 \\
\text { mortes por mesotelioma na Grã Betanha }\end{array}$ \\
\hline $\begin{array}{l}\text { 11. Murlidhar } \\
\text { V, KanhereV. } \\
\text { 2005/EUA }\end{array}$ & $\begin{array}{l}\text { Objetivo: Identificar trabalhadores } \\
\text { que sofrem com asbestose em } \\
\text { uma empresa, avaliando suas } \\
\text { incapacidades. } \\
\text { Delineamento: Estudo caso controle } \\
\mathrm{N}=181\end{array}$ & IV & $\begin{array}{l}\text { Trabalhadores na Índia: } 22 \% \text { com } \\
\text { asbestose, por exposição de até } 20 \text { anos } \\
\text { ao amianto. Incapacidades apresentadas: } \\
34 \% \text { com taxas inspiratórias basais em } \\
\text { atraso; } 82 \% \text { com volume expiratório } \\
\text { forçado acima de } 80 \% \text { no primeiro } \\
\text { segundo e capacidade de força vital } \\
\text { forçada; } 66 \% \text { com capacidade de força } \\
\text { inferior a } 80 \% \text { do valor previsto, em } \\
\text { avaliação radiológica; } 7 \% \text { com doença } \\
\text { pleural; } 10 \% \text { tanto doença pleural e em } \\
\text { Parênquima. }\end{array}$ \\
\hline
\end{tabular}




\begin{tabular}{|c|c|c|c|}
\hline $\begin{array}{l}\text { 12. Delgermaa } \\
\text { V, Takahashi K, } \\
\text { Park EK, e Le } \\
\text { GV, Hara T, } \\
\text { Sarahan T.(32) } \\
\text { 2011/ Suíça }\end{array}$ & $\begin{array}{l}\text { Objetivo: Realizar analise descritiva } \\
\text { das mortes por mesotelioma relatados } \\
\text { mundialmente entre } 1994 / 2008 \\
\text { Delineamento: Estudo descritivo }\end{array}$ & $\mathrm{VI}$ & $\begin{array}{l}\text { Em } 83 \text { países foram } 92.253 \text { mortes por } \\
\text { mesolteioma; Doença por localização: } \\
\text { pleura } 41,3 \% \text {; peritônio } 4,5 \% \text {; pericárdio } \\
0,3 \% \text {; locais não especificados } 43,1 \% \text {. }\end{array}$ \\
\hline $\begin{array}{l}\text { 13. Tan E, } \\
\text { Warren N, } \\
\text { Darnton AJ, } \\
\text { Hodgson JT. (33) } \\
\text { 2010/ Inglaterra }\end{array}$ & $\begin{array}{l}\text { Objetivo: Avaliar prospectivamente } \\
\text { as previsões de mortalidade por } \\
\text { mesotelioma na Grã Betanha. } \\
\text { Delineamento: Estudo de coorte. }\end{array}$ & IV & $\begin{array}{l}\text { Prospectivamente o número estimado } \\
\text { de casos: 1,08 casos por milhão de } \\
\text { habitantes. Mortalidade entre os homems } \\
\text { prevista para 2016: } 2.040 \text { mortes. Período } \\
\text { de } 1968 \text { a 2050: previsão de } 91.000 \\
\text { mortes. }\end{array}$ \\
\hline $\begin{array}{l}\text { 14. Ferrante } \\
\text { D, Bertolotti } \\
\text { M, Todesco } \\
\text { A, Mirabelli } \\
\text { D, Terracini B, } \\
\text { Magnani C. }{ }^{(34)} \\
\text { 2007/ EUA }\end{array}$ & $\begin{array}{l}\text { Objetivo: Analisar a incidência de } \\
\text { mortalidade por Mesotelioma em } \\
\text { esposas de trabalhadores expostos } \\
\text { ao amianto entre } 1907 \text { e } 1986 \text { na Itália. } \\
\text { Delineamento: Estudo de coorte. }\end{array}$ & IV & $\begin{array}{l}\text { Mortalidade: } 11 \text { casos de mesotelioma } \\
\text { pleural em esposas por exposição } \\
\text { doméstica ao amianto }\end{array}$ \\
\hline $\begin{array}{l}\text { 15. Clemente } \\
\text { M, Reig-Botella } \\
\text { A, Prados JC. (35) } \\
\text { 2015/ Brasil }\end{array}$ & $\begin{array}{l}\text { Objetivo: Avaliar o estado de } \\
\text { saúde psicossocial e mental dos } \\
\text { profissionais na Espanha afetados } \\
\text { pelo amianto } \\
\text { Delineamento: Estudo transversal } \\
\mathrm{N}=110\end{array}$ & VI & $\begin{array}{l}\mathrm{Na} \text { avaliação da saúde psicossocial e } \\
\text { mental dos profissionais apresentaram: } \\
\text { doenças mentais, transtorno obsessivo } \\
\text { compulsivo, ansiedade, depressão, } \\
\text { fobias, paranoia, psicose, sensibilidade } \\
\text { interpessoal, hostilidade, gravidade } \\
\text { global }\end{array}$ \\
\hline
\end{tabular}

\section{- DISCUSSÃO}

Os estudos apresentam o impacto causado na população adulta pela exposição ao amianto. Cerca de 200 milhões de toneladas de amianto são comercializadas mundialmente, e desde a extração do mineral até a manufatura dos produtos representam perigo à saúde da população(10) .

O desenvolvimento de doenças pela exposição ao amianto teve prevalência para o mesotelioma maligno e sua mortalidade ${ }^{(12,16,18,21,23-25)}$. Esta patologia tem relação de 80 a $90 \%$ com a exposição ao amianto $^{(12)}$. No estudo realizado na Espanha em 2013, os índices de mortalidade causada pelo câncer pleural e mesotelioma aumentou no decorrer das décadas, com prevalência para o sexo masculino. A comercialização foi proibida no país desde o ano de $2002^{(21)}$.

O adoecimento no sexo masculino por mesotelioma é resultante das funções ocupacionais lideradas por homens, portanto a carga de doenças amianto relacionadas tendem a prevalecer. A exposição mesmo por um período de tempo reduzido é uma ameaça à saúde ${ }^{(9)}$. O gênero masculino apresentam risco acentuado de desenvolver doenças provenientes da exposição, devido ao fato dos homens estarem à frente dos cargos ocupacionais nas empresas que manipulam o amianto ${ }^{(32)}$.

Mesmo que o amianto tenha sido proibido em determinadas regiões, os casos de mesotelioma continuam prospectivamente, resultante do período de latência, que pode chegar a 50 anos após o fim da exposição ${ }^{(36)}$. Um exemplo é o Japão, que em 2050 poderá atingir 66.327 mortes, devido ao período de latência ${ }^{(25)}$, corroborado com estudo na Grã Betanha, que estimou para o país cerca de 90.000 mortes para o mesmo ano ${ }^{(30)}$.

Nesta perspectiva a associação do mesotelioma e mortalidade apresentam relação intensa nos estudos $^{(1,25,27,30,32-34)}$. O exemplo do estudo realizado em 2010 na Grã-Bretanha estimou 2.040 casos de mortes por mesotelioma para o ano de 2016, e nesta região as mortes poderão ocorrer até o ano de $2050^{(33)}$. Estudos estão sendo realizados para detecção precoce e tratamento para melhores resultados no mesotelioma ${ }^{(37)}$. O diagnóstico tardio torna o prognostico ruim, com média de sobrevida de 12 
meses $^{(36)}$.

A estimativa de mesotelioma não mostra sinais de diminuição e as taxas de incidência mais elevada são descritas em alguns países da Europa (Reino Unido, Países Baixos, Malta, Bélgica) e na Oceania (Austrália e Nova Zelândia). Observa-se que a subnotificação da doença determina a falta de dados mundiais e não permite assim que a consciência dos riscos seja atribuída ao amianto ${ }^{(38)}$. Neste sentido, os casos de mortes por mesotelioma ocorridos mundialmente no período de 1994 e 2008 apontam as subnotificações da doença como um problema e revelam que o Brasil é um dos países que apresentam inconsistência de notificações referentes ao adoecimento por mesotelioma ${ }^{(32)}$. Um dado preocupante, pois o Brasil ocupa o terceiro lugar na lista de maior produtor do amianto ${ }^{(17)}$.

No Japão, a comercialização do amianto foi proibida desde $2004^{(19)}$, entretanto um estudo realizado no país em 2012 estimou que de 2003 a 2050 pode chegar a 66.327 mortes de indivíduos com idade entre 50 e 89 anos $^{(25)}$. Em outras regiões, a média é de 70 anos e no sexo masculino ${ }^{(24)}$.

Os riscos do amianto se estendem à população em geral e não somente a trabalhadores, inclusive decorrentes de fenômenos naturais, como em um terremoto, que expõe o amianto presente nos materiais de construção, também é considerado um fator de risco à população ${ }^{(25)}$. Os riscos ambientais conferem igual preocupação por não existirem níveis seguros de exposição ${ }^{(39)}$.

Outra patologia amianto relacionada com mortalidade acentuada é a asbestose com 5.396 mortes $^{(27)}$. A doença é definida como fibrose pulmonar intersticial difusa, atribuída à inalação de fibras de amianto $^{(40)}$. Complicações broncopulmonares e morbidades como insuficiência cardíaca também apresentam relações com o amianto ${ }^{(27)}$.

Na Itália, objetivando identificar as taxas de mortalidade de 1247 trabalhadores de uma fábrica produtora do cimento localizada na cidade de Nápoles, observou-se um aumento da mortalidade por doenças respiratórias, em particular a pneumoconiose, asbestose, câncer pulmonar, peritoneal e aumento pouco significativo para câncer de reto ${ }^{(23)}$. Neste país o uso do amianto foi proibido em $1992^{(18)}$.

Os países que proibiram o uso do amianto são os que possuem as maiores taxas de mortalidade mundiais, devido ao uso por décadas ${ }^{(41)}$. Isso se deve ao fato do aumento da exportação e da produção de materiais com o amianto, principalmente no período pós-guerra, gerando o desenvolvimento econômico de diversos países ${ }^{(21,25)}$.

$\mathrm{Na}$ Espanha os trabalhadores de uma empresa de aço, expostos ao amianto crisotila direta e indiretamente, com jornada de trabalho de 8 a 14 horas por dia e média de 21 anos de exposição, adoeceram por asbestose, placas pleuras, mesotelioma e câncer de pulmão, com prevalência para o sexo masculino e média de idade de 65 anos $^{(22)}$.

A crisotila é responsável por mais de $90 \%$ do amianto utilizado mundialmente ${ }^{(42)}$ e há a asbestose e os cânceres associados, tais como: câncer de pulmão, laringe, ovários, mesotelioma ${ }^{(6)}$. Assim como pode ser observado na Carolina do Norte EUA, onde 3002 trabalhadores foram acompanhados por um mês, em contato com a crisotilae, concluíram que a ocorrência de câncer de pulmão variou de acordo com a acumulação da fibra ${ }^{(29)}$.

O impacto do amianto ultrapassa os aspectos biológicos, trazendo alterações psicoemocionais significativas. Estudos recentes na Espanha referem que alterações psicoemocionais como somatização, transtornos obsessivos compulsivos, depressão, paranoia, ansiedade fóbica, foram encontrados em trabalhadores afetados pelo amianto ${ }^{(35)}$. Faz-se necessário o reconhecimento como enfermidade ocupacional para as doenças amianto relacionadas, bem como o pagamento de indenizações e o diagnóstico precoce ${ }^{(28)}$.

É importante observar o risco potencial para a exposição ao amianto fora do ambiente ocupacional(6). Em um estudo mulheres casadas com trabalhadores diretamente expostos ao amianto apresentaram incidência de mesotelioma e aumento significativo para neoplasias malignas do sistema respiratório confirmando o impacto na saúde na saúde do familiar devido ao contato com a fibra de amianto em roupas e objetos de trabalhadores ${ }^{(34)}$.

Os estudos concluem que não há níveis seguros de exposição, mesmo por um período curto 
de tempo ${ }^{(10)}$. Na Itália, foram acompanhados trabalhadores por um mês em uma empresa líder em manipulação de amianto entre os anos de 1946 e 1984, evidenciou o adoecimento por doenças pleurais, peritoneais, confirmando o desenvolvimento de doenças independente do tempo de exposição(26).

Os estudos retrospectivos quanto prospectivos alertam que a mortalidade por doenças provocadas pela exposição do amianto tende a números alarmantes, configurando-se em um problema de saúde pública em nível mundial.

\section{CONSIDERAÇÕES FINAIS}

O impacto do amianto na saúde do adulto ficou caracterizado pelo desenvolvimento de doenças com altas taxas de mortalidade e prevalência do mesotelioma maligno; no adoecimento resultante da exposição ocupacional para o trabalhador do sexo masculino e, em familiares, principalmente nas mulheres, esposas desses trabalhadores e na exposição ambiental; o longo período de latência para o desenvolvimento de doenças amianto relacionadas, com implicações para o diagnóstico precoce. A produção de conhecimentos e o debate sobre o impacto ganha força no campo da saúde do trabalhador e na saúde da população em geral sobre a necessidade do banimento de seu uso nos países que o mantém em escala industrial e comercial. Mesmo com a comprovação de doenças provocadas pelo amianto, países como o Brasil permanecem na produção e consumo sem uma legislação que proíba seu uso, por questões econômicas.

A proibição do amianto em âmbito mundial será possível com a substituição da fibra na manufatura de produtos por outros materiais, através do desenvolvimento de mecanismos tecnológicos e econômicos. As pesquisas demonstram de maneira enfática que não há forma segura de trabalhar com produtos que contenham amianto.

Atualmente a OMS recomenda ações para a prevenção de doenças relacionadas à exposição ao amianto, para otimizar o diagnóstico precoce, estabelecer o registro de pessoas expostas e o acompanhamento nos serviços de saúde dos portadores de doenças amianto relacionadas. $\mathrm{O}$ adoecimento por mesotelioma maligno conferiu prevalência significativa para o estudo. A taxa mortalidade é preocupante, haja vista que as possibilidades terapêuticas são limitadas.

Destacamos a importância de ampliar a produção científica nacional sobre a temática. O Brasil ocupa o terceiro lugar no ranking de produtor mundial do amianto e faltam estudos retrospectivos e prospectivos sobre o impacto na saúde da população adulta para a definição de monitoramento e controle da população exposta.

\section{REFERÊNCIAS}

1. Kim SY, Kim YC, Kim Y, Hong WH. Predicting the mortality from asbestos-related diseases based on the amount of asbestos used and the effects of slate buildings in Korea. Sci Total Environ.[Internet] 2016; 542(PtA) [acesso em 15 mar 2016]. Disponível: https://dx.doi.org/10.1016/j.scitotenv.2015.10.115

2. International Agency Research Cancer (IARC). Monographs Asbestos (chrysotile, amosite, crocidolite, tremolite, actinolite, and anthophyllite) [Internet] 2012 [acesso em 10 mar 2016]. Disponível: http://monographs. iarc.fr/ENG/Monographs/vol100C/mono100C-11.pdf

3. Doll R. Mortality from lung cancer in asbestos workers. Br J Ind Med. [Internet] 1955; 12. [acesso em 10 mar 2016] Disponivel: http://www.ncbi.nlm.nih.gov/pmc/articles/PMC1035472/pdf/brjindmed00006-0005.pdf

4.Wagner JC, Sleggs CA, Marchand P. Difuse pleural mesothelioma and asbestos exposure in the north western cape province. Brit. J. Industr. Med. [Internet] 1970; 17(270) [acesso em 10 mar 2016]. Disponível:

http://www.ncbi.nlm.nih.gov/pmc/articles/PMC1038078/pdf/brjindmed00208-0014.pdf

5.Rong Y, Luo X, Zhang Z, Cui X, Liu Y, Chen W. Occupational exposure to asbestos and cardiovascular related diseases: a meta-analysis. Prev. Med Rep. [Internet] 2015; 2 [acesso em 10 mar 2016]. Disponível:

https://dx.doi.org/10.1016/j.pmedr.2015.10.005 
6.Word Health Organization (WHO). Chrysotile Asbestos. 2014 [acesso em 10 mar 2016]. Disponível: http://www.who.int/ipcs/assessment/public_health/chrysotile_asbestos_summary.pdf

7. Scimeca M, Pietroiusti A, Milano F, Anemona L, Orlandi A, Marsella LT, et al. Elemental analysis of histological specimens: a method to um mask nano asbestos fibers. Eur J Histochem. [Internet] 2016; 60(1) [acesso 07 mai 2015]. Disponível: https://dx.doi.org/10.4081/ejh.2016.2573

8. Marchiori L, Marangi G, Ballarin N, Valentini F, D'Anna M, Barbina P, et al. Proposal of an Italian national protocol of health surveillance for former asbestos workers: anongoing Project. Epidemiol Prev. [Internet] 2016; 40(1Suppl 1) [aceso em 28 abr 2016]. Disponível: https://dx.doi.org/10.19191/EP16.1S1.P068.033

9. Lin CK, Chang YY, Wang JD, Lee LJH. Increased standardised incidence ratio of malignant pleural mesothelioma in Taiwanese asbestos workers: a 29 year retrospective cohort study. Biomed Res Int. [Internet] 2015; 2015(2015) [acesso em 18 abr 2016] Disponível: http://dx.doi.org/10.1155/2015/678598

10. Frank AL, Joshi TK. The global spread of asbestos. Ann Glob Health. [Internet] 2014; 80(4) [acesso em 15 mar 2016]. Disponível: https://dx.doi.org/10.1016/j.aogh.2014.09.016

11. Baur X, Soskolne CL, Lemen R, Schneider J, Woitowitz HS, Budnik LT. How conflicted authors undermine the World Health Organization (WHO) campaign to stop all use of asbestos: spotlight on studies showing that chrysotile is carcinogenic and facilitates other non-cancer asbestos-related diseases. Int J Occup Environ Health. [Internet] 2015; 21(2) [acesso em 15 mar 2016]. Disponível: https://dx.doi.org/10.1179/2049396714Y.0000000105

12. Baas P, Burgers S. ASIA: asbestos stop in Asia. Respirology. [Internet] 2015; 20(4) [acesso em 10 mar 2016$].$ Disponível: https://dx.doi.org/10.1111/resp.12533

13. Barber CM, Wiggans RE, Young C. UK asbestos imports and mortality due to idiopathic pulmonary fibrosis. Occup Med. [Internet] 2016; 66(2) [acesso em 28 mar 2016]. Disponível: https://dx.doi.org/10.1093/occmed/kqv142

14.Tiwari RR, Saha A. Knowledge and attitude towards asbestos hazards among asbestos work $\neg$ ers in India. Int J Occup Environ Med. [Internet] 2015; 6(1) [acesso em 28 mar 2016]. Disponível:

http://www.theijoem.com/ijoem/index.php/ijoem/article/view/465/575

15. Assis LD, Isoldi MC. The development of MM is strongly correlated with exposure to asbestos and erionite, as well as tosimianvirus 40. J Bras Pneumol. [Internet] 2014; 40(5) [acesso em 28 mar 2016]. Disponível:

https://dx.doi.org/10.1590/S1806-37132014000500018

16. Kameda T, Ken T, Rocko Kim, Yin J, Movahed M, Park EK, et al. Asbestos: use, bans and disease burden in Europe. Bulletin of the World Health Organization. [Internet] 2014; 92 [acesso em 28 mar 2016]. Disponível: http://dx.doi.org/10.2471/BLT.13.132118

17.Sen D. Working with asbestos and the possible health risks. Occup Med. [Internet] 2015; 65(1) [acesso em 15 mar 2016]. Disponível: https://dx.doi.org/10.1093/occmed/kqu175

18.International Ban Asbestos Secretariat. Current asbestos ban and restrictions. 2015 [acesso em 28 mar 2016 ]. Disponível: http://ibasecretariat.org/alpha_ban_list.php

19.Ganong LH. Integrative reviews of nursing research. Res Nurs Health. [Internet] 1987; [acesso em 15 mar 2016] 10(1): 1-11. Disponível:

https://www.researchgate.net/publication/19518297_Integrative_review_of_nursing_reseach

20. Agency for healthcare research and quality (AHRQ). Methods Guide for Comparative Effectiveness Reviews. 2012 [acesso 09 mar 2016]. Disponível: https://www.effectivehealthcare.ahrq.gov/ehc/products/457/1752/ methods-guidance-grading-evidence-131118.pdf

21. Abente GL, Gómez MG, Navarro AM, Navarro PF, Ramis R, Perez JG, et al. Pleural cancer mortality in Spain: time-trends and updating of predictions up to 2020. BMC Cancer. [Internet] 2013; 13(528) [acesso em 15 mar 2016]. Disponível: https://dx.doi.org/10.1186/1471-2407-13-528

22. Zurbriggen R, Capone L. Enfermedad pulmonar por amianto em trabajadores deacería. Medicina. [Internet] 2013; 73(3) [acesso em 15 mar 2016]. Disponível: http://www.scielo.org.ar/scielo.php?script=sci_arttext\&pid $=$ S0025-76802013000300004 
23. Menegozzo S, Comba P, Ferrante D, SantisM, Gorini G, Izzo F, et al. Mortality study in an asbestos cement factory in Naples, Italy. Ann Ist Super Sanità. [Internet] 2011; 47(3) [acesso 15 mar 2016]. Disponível:

https://dx.doi.org/0.4415/ann_11_03_10

24.Wang X, Yano E, Lin S, Yu ITS, Lan Y, Tse LA, et al. Cancer mortality in Chinese chrysotile asbestos miners: exposure-response relationships. PLoSONE. [Internet] 2013; 8(8) [acesso em 15 mar 2016]. Disponível: http:// dx.doi.org/10.1371/journal.pone.0071899

25. Myogin T, Azuma K, Okumura J, Uchiyama I. Future Trends of Mesothelioma Mortality in Japan Based on a Risk Function. Industrial Health. [Internet] 2012; 50 [acesso em 15 mar 2016]. Disponível:

https://www.jstage.jst.go.jp/article/indhealth/50/3/50_MS1184/_pdf

26. Pira E, Pelucchi C, Buffoni L, Palmas A, Turbiglio M, Negri E, et al. Cancer mortality in a Cohort of asbestos textile workers. Br J Cancer. [Internet] 2005; 92(3) [acesso em 10 mar 2016]. Disponível:

https://dx.doi.org/10.1038/sj.bjc.6602240

27.Darnton A, Hodgson J, Benson P, Coggon D. Mortality from Asbestosis and Mesothelioma in Britain by Birth Cohort. Occup Med (Lond). [Internet] 2012; 62(3) [acesso em 15 mar 2016]. Disponível:

https://dx.doi.org/10.1093/occmed/kqs119

28.Pietro MA, Suess A, March JC, Danet A, Corral OP, Martin A. Opiniones y expectativas de pacientes con enfermidades relacionadas com La exposición al amianto. An Sist Sanit Navar. [Internet] 2011; 34(1) [acesso em 16 fev 2016]. Disponível: http://recyt.fecyt.es/index.php/ASSN/article/view/11147/8014

29. Cole SR, Richardson DB, Haitão C, Naimi A. Analysis of occupational asbestos exposure and lung cancer mortality using the $\mathrm{g}$ formula. Am J of Epidemiol. [Internet] 2013; 177(9) [acesso em 16 fev 2016]. Disponível: https://dx.doi.org/10.1093/aje/kws343

30. Hodgson JT, McElvenny DM, Darnton AJ, Price MJ, Peto J. The Expected burden of mesothelioma mortality in Great Britain from 2002 to 2050. Br J Cancer. [Internet] 2005; 92 [acesso em 10 fev 2016]. Disponível:

http://www.ncbi.nlm.nih.gov/pmc/articles/PMC2362088/pdf/92-6602307a.pdf

31. Murlidhar V, KanhereV. Asbestosis in an asbestos composite mill at Mumbai: A prevalence study. Environ health. [Internet] 2005; 4(24) [acesso em 16 fev 2016]. Disponível: https:dx.doi.org/10.1186/1476-069X-4-24

32. Delgermaa V, Takahashi K, Park EK, Le GV, Hara T, Sarahan T. Global mesothelioma deaths reported to the World Health Organization between 1994 and 2008. Bull World Health Organ. [Internet] 2011; 89(10) [acesso em 18 fev 2016]. Disponível: https://dx.doi.org/10.2471/BLT.11.086678

33. Tan E, Warren N, Darnton AJ, Hodgson JT. Projection of mesothelioma mortality in Britain using Bayesian methods. Br J Cancer. [Internet] 2010; 103(3) [acesso em 18 fev 2016]. Disponível:

https://dx.doi.org/10.1038/sj.bjc.6605781

34.Ferrante D, Bertolotti M, Todesco A, Mirabelli D, Terracini B, Magnani C. Cancer Mortality and Incidence of Mesothelioma in a Cohort of Wives of Asbestos Workers in Casale Monferrato, Italy. Environ Health Perspect. [Internet] 2007; 115(10) [acesso em 19 fev 2016]. Disponível: https://dx.doi.org/10.1289/ehp.10195

35. Clemente M, Reig-Botella A, Prados JC. Alterations in psychosocial health of people affected by asbestos poisoning. Rev Saúde Públic.[Internet] 2015; 49(24) [acesso em 19 mar 2016]. Disponível:

http://www.ncbi.nlm.nih.gov/pmc/articles/PMC4390070/

36. Neumann V, Loseke S, Nowak D, Hert FJ, Tannapfel A. Malignant Pleural Mesothelioma: Incidence, Etiology, diagnosis, treatment, and occupational health. Dtsch Arztebl Int. [Internet] 2013; 110 (18) [acesso em 02 abril 2016]. Disponível: https://dx.doi.org/10.3238/arztebl.2013.0319

37. Jing XQ, Zhoul L, Sun XD, YU JM, Meng X. Premetrexed maintenance therapy following bevacizumab containing first line chemoterapy in advanced malignant pleural mesothelioma: a case report and literature review. Medicine. [Internet] 2016; 95(14) [acesso em 02 abril 2016]. Disponível:

https://dx.doi.org/10.1097/MD.0000000000003351

38. Bianchi C, Bianchi T. Global Mesothelioma epidemic: Trends and Features. Indian J Occup Environ Med. [Internet] 2014; 18(2) [acesso em 02 abril 2016]. Disponível: https://dx.doi.org/10.4103/0019-5278.146897 
39. Collegium Ramazzini (CR). The global health dimensions of asbestos and asbestos related diseases. J Occup Health. 2016 [acesso em 02 abril 2016]. Disponível:

https://www.jstage.jst.go.jp/article/joh/advpub/0/advpub_16-2002-ST/_pdf

40. Lee EK, Kim JS, Kim Y, Park JS. CT findings in people who were environmentally exposed to asbestos in korea. J Korean Med Sci. [Internet] 2015; 30(12) [acesso em 03 abril 2016]. Disponível:

https://dx.doi.org/10.3346/jkms.2015.30.12.1896

41. Kameda T,Takahashi K, Rokho K, Jiang Y, Movahed M, Park EK, et al. Asbestos: use, bans and disease burden in Europe. Bull World Health Organ. [Internet] 2014; 92(11) [acesso em 05 abril 2016]. Disponível:

https://dx.doi.org/10.2471/BLT.13.132118

42. Gulino GR, Polimeni M, Prato M, Gazzano E, Kopecka J, Colombatto S, et al. Effects of crysotile exposure in human bronchial epithelial cells: insights on the pathogenic mechanism of asbestos related diseases. Environ Health Perspect. [Internet] 2015; 18 [acesso em 05 abril 2016]. Disponível: https://dx.doi.org/10.1289/ehp.1409627 